\title{
Remoção de algas em um sistema de biofiltros submersos
}

\author{
Removal of algae in a submerse biofilter system
}

Gerson Pavanelli** ${ }^{*}$, Eduardo Cleto Pires ${ }^{1} \mathbb{C}$

口-

\section{RESUMO}

Esta pesquisa propôs o desenvolvimento de um sistema de biofiltros submersos (SBS), modificados com base na configuração de filtros de pedra, visando ao póstratamento do efluente de lagoas de estabilização, inicialmente para a remoção de algas. Foram implantados 2 conjuntos de biofiltros submersos para captar esgoto tratado de duas diferentes profundidades de uma lagoa de maturação - a 60 cm (em uma zona dessa lagoa chamada superficial - ZS) e a 180 cm (em outra zona dessa lagoa chamada intermediária - ZI). Foi utilizada, como recheio dos biofiltros submersos, pedra brita no 3 , nas seguintes alturas de leito filtrante: 50, 100, 150 e 200 cm, tendo por objetivo avaliar a influência desse fator sobre a eficiência de remoção de algas mediante análise de clorofila A e outras 16 variáveis de qualidade associadas neste estudo. Obtiveram-se eficiências para demanda química de oxigênio (DQO) no intervalo de 65 a 80\% para ZS e, para Zl, os resultados dessa eficiência tiveram muita variação, sem estabilidade - de forma prática, portanto, o ponto de coleta na ZI mostrou-se não recomendado. Quando às eficiências para clorofila A, para ZS, o intervalo alcançado variou de 65 a 99\% e, para ZI, esses resultados foram inferiores aos da ZS. Concluiu-se quea extração a partir da zona superficial da lagoa de maturação e uma profundidade de leito entre 150 e 200 cm foram os parâmetros operacionais que levaram a um melhor desempenho global dos biofiltros submersos modificados e, mais especificamente, quanto às seguintes variáveis: DQO, demanda bioquímica de oxigênio (DBO), clorofila A e sólidos totais.

Palavras-chave: biofiltros; pós-tratamento de lagoa; remoção de algas.

\begin{abstract}
This research proposed the development of a submerged biofilters system, modified based on the configuration of rock filters, aiming at the effluent post-treatment of stabilization lagoons, initially for algae removal. Two sets of submerged biofilters were implemented to collect treated sewage from two different depths of a maturation lagoon - at $60 \mathrm{~cm}$ (in a zone of this lagoon called superficial-SZ) and at $180 \mathrm{~cm}$ (in a zone of this lagoon called intermediary-IZ). It was used, as filling of submerged biofilters, gravel No. 3, at filtering layers heights of: 50, 100, 150 and $200 \mathrm{~cm}$, aiming to evaluate the influence of this factor over algae removal efficiency toward chlorophyll $a$, and other 16 quality variables associated in this study. For SZ, efficiencies were obtained for COD in the range of 65 to $80 \%$, and for $I Z$ the efficiency results had a lot of variation, without stability, in a practical way, so the collection point in ZI was not recommended. For SZ efficiencies were obtained for chlorophyll $a$ in the range of 65 to $99 \%$, and for IZ the efficiency results were lower than those of SZ. It was concluded that the collecting from superficial zone of the maturation lagoon, and a layer depth between 150 and $200 \mathrm{~cm}$, were the operational parameters that led to a better overall performance of the modified submerged biofilters, and more particularly to: COD (Chemical Oxygen Demand), BOD (Biochemical Oxygen Demand), chlorophyll a, and total solids.
\end{abstract}

Keywords: biofilter; lagoon post treatment; algae removal.

\section{INTRODUÇÃO}

Entre os principais problemas enfrentados em estações de tratamento de esgoto (ETE) providas de sistemas de tratamento por meio de lagoas, destaca-se o excesso de produção de algas, concorrendo para o não atendimento aos requisitos das legislações ambientais federal e estadual, para efluentes lançados em cursos d'água, no que diz respeito, por exemplo, aos valores de nitrogênio amoniacal.

Tal fato tem sido recorrente em instalações situadas em todo o mundo, ocorrendo também, nos últimos anos, em diversos sistemas

de tratamento de esgoto da Companhia de Saneamento do Paraná (SANEPAR), o que motivou o interesse na presente pesquisa, visando encontrar soluções para o problema enfrentado.

Para tanto, foi desenvolvida uma nova configuração de biofiltro submerso, modificado a partir da configuração de filtros de pedra, objetivando o pós-tratamento do efluente de uma lagoa de maturação, localizada no interior das instalações da ETE Norte da SANEPAR.

Nesse local, foram implantados 2 conjuntos de biofiltros submersos para captar esgoto tratado de duas diferentes profundidades dessa

\section{口-}

'Universidade de São Paulo - São Carlos (SP), Brasil.

*Autor correspondente: sanetec@sanetec.com.br

Recebido: 26/06/2014 - Aceito: 10/09/2018 - Reg. ABES: 136913 
lagoa - a $60 \mathrm{~cm}$ (em uma zona chamada superficial - ZS) e a $180 \mathrm{~cm}$ (em outra zona chamada intermediária - ZI).

Para a análise da eficiência de remoção das algas por parte do sistema de biofiltros submersos (SBS), foi utilizada a determinação analítica de clorofila A, de modo que se quantificasse indiretamente a presença de algas no efluente dos biofiltros submersos e se avaliasse qual a espessura de camada filtrante mais adequada para essa finalidade.

\section{METODOLOGIA}

As instalações de biofiltros submersos foram construídas próximas ao acesso da ETE Norte, com as tomadas da ZS $(60 \mathrm{~cm})$ e da ZI $(180 \mathrm{~cm})$, na parte final da lagoa de maturação dessa estação, sendo recalcado o afluente aos biofiltros submersos por meio de bombas submersíveis. Para efeito de apresentação do sítio experimental onde a presente pesquisa foi desenvolvida, é apresentada, na Figura 1, a planta de situação e o respectivo perfil hidráulico da estação, em Cascavel (PR). A Tabela 1 apresenta os parâmetros do projeto dessa estação (conforme dados da SANEPAR).

Nas tomadas na lagoa, efetuadas por meio de duas bombas submersíveis, foi considerado que ocorre maior concentração de algas próximo à superfície, sendo assim, foram estudadas duas alturas diferentes como afluentes aos biofiltros submersos, uma a $0,60 \mathrm{~m}$ de profundidade da superfície da lagoa e outra a 1,80 m.

A Figura 2 apresenta diferentes vistas das instalações experimentais em sua fase final de implantação na ETE Norte da SANEPAR em Cascavel (PR).

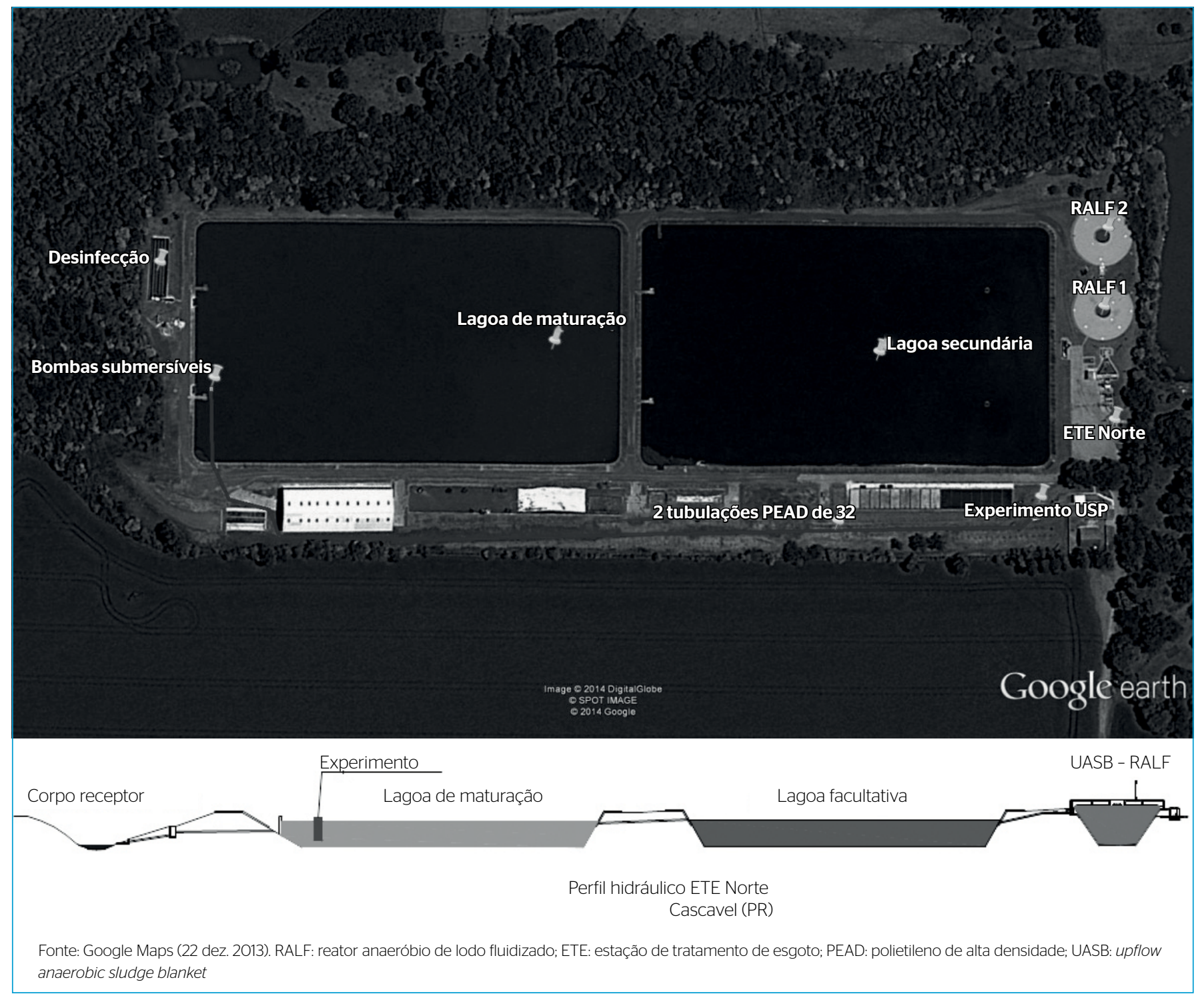

Figura 1 - Planta e perfil da estação de tratamento de esgoto Norte da Companhia de Saneamento do Paraná, situada em Cascavel (PR), com seu respectivo perfil hidráulico. 


\section{Sistema de biofiltros submersos}

Foram construídos quatro biofiltros submersos para a alimentação de afluente da ZS e mais quatro para a da ZI da lagoa de maturação. A Tabela 2 mostra as profundidades de camada filtrante de cada um desses biofiltros.

Para a execução dos biofiltros submersos, foram utilizados, como fôrma, tubos da TIGRE-ADS ${ }^{\circ}$ em polietileno de alta densidade (PEAD) com diâmetro nominal (DN) de $1.050 \mathrm{~mm}$ e extensão de $6 \mathrm{~m}$. Como a utilização dos biofiltros submersos deveria ocorrer na vertical (Figura 3), para o tamponamento da base, executou-se um revestimento em fibra de vidro, internamente, em todo o tubo, para garantir a estanqueidade. Foram deixados orifícios, um para o afluente, outro para o efluente do biofiltro submerso em policloreto de polivinila (PVC) com DN de $25 \mathrm{~mm}$ e a saída para descarga em PVC com DN de $100 \mathrm{~mm}$.
Os biofiltros submersos foram preenchidos com 4 alturas de recheio: 50, 100, 150 e $200 \mathrm{~cm}$. Para os recheios, foi utilizada pedra brita $\mathrm{n}^{\circ} 3$ (observar o corte do biofiltro submerso - FS - na Figura 4). Foi necessário realizar uma seleção dessas pedras, utilizando o dobro do volume previsto, para a obtenção de leitos filtrantes homogêneos.

Tabela 2 - Altura da camada filtrante para cada biofiltro submerso.

\begin{tabular}{l|c|c|c}
$\begin{array}{l}\text { N. do biofiltro } \\
\text { submerso (ZI) }\end{array}$ & $\begin{array}{c}\text { Altura da cama- } \\
\text { da filtrante }(\mathrm{cm})\end{array}$ & $\begin{array}{c}\text { N. do biofiltro } \\
\text { Submerso }(\text { ZS) }\end{array}$ & $\begin{array}{c}\text { Altura da cama- } \\
\text { da filtrante }(\mathrm{cm})\end{array}$ \\
\hline BS-1 & 50 & BS 5 & 50 \\
\hline BS-2 & 100 & BS 6 & 100 \\
\hline BS-3 & 150 & BS 7 & 150 \\
\hline BS-4 & 200 & BS 8 & 200 \\
\hline
\end{tabular}

ZI: zona intermediária; ZS: zona superficial; BS: biofiltro submerso.

Tabela 1 - Parâmetros de projeto da estação de tratamento de esgoto Norte de Cascavel (PR) (conforme dados da Companhia de Saneamento do Paraná).

\begin{tabular}{|c|c|c|c|c|}
\hline PARÂMETRO & $\begin{array}{c}\text { Ano } 2000 \\
\text { pa }^{\mathrm{a}} \text { etapa }\end{array}$ & $\begin{array}{c}\text { Ano } 2010 \\
2^{\mathrm{a}} \text { etapa }\end{array}$ & Ano 2020 & $\begin{array}{l}\text { Saturação } \\
\text { Fim de Plano }\end{array}$ \\
\hline População atendida (hab.) & 46.628 & 70.950 & 94.983 & 144.366 \\
\hline Vazão doméstica média $\left(L . S^{-1}\right)$ & 69,22 & 105,58 & 141,67 & 209,25 \\
\hline Vazão de infiltração em épocas de chuvas $\left(L . S^{-1}\right)$ & 69,06 & 70.05 & 70,05 & 80,28 \\
\hline Vazão média sanitária $\left(\overline{\mathrm{Q}}=\mathrm{L} . \mathrm{S}^{-1}\right)$ & 138,28 & 175,63 & 211,72 & 290,11 \\
\hline Vazão do dia de maior contribuição (L.S $\left.{ }^{-1}\right)$ & 152,13 & 196,74 & 240,05 & 331,96 \\
\hline Vazão máxima (L.S'ㄱ) & 193,66 & 260,09 & 325,05 & 457,51 \\
\hline Carga DBO (kg.dia') & 2.518 & 3.831 & 5.129 & 7.796 \\
\hline Concentração DBO (mg. $\left.\mathrm{L}^{-1}\right)$ & 211 & 252 & 280 & 311 \\
\hline Carga DQO (kg.dia'-1) & 5.036 & 7.662 & 10.258 & 15.592 \\
\hline Concentração DQO (mg. L'1) $\left.^{-1}\right)$ & 422 & 504 & 560 & 622 \\
\hline NMP coliforme fecal.100 ml-1 (sem chuvas) & $3,9 \times 10^{7}$ & $4,7 \times 10^{7}$ & $5,2 \times 10^{7}$ & $5,8 \times 10^{7}$ \\
\hline
\end{tabular}

DBO: demanda bioquímica de oxigênio; DQO: demanda química de oxigênio; NMP: número mais provável.

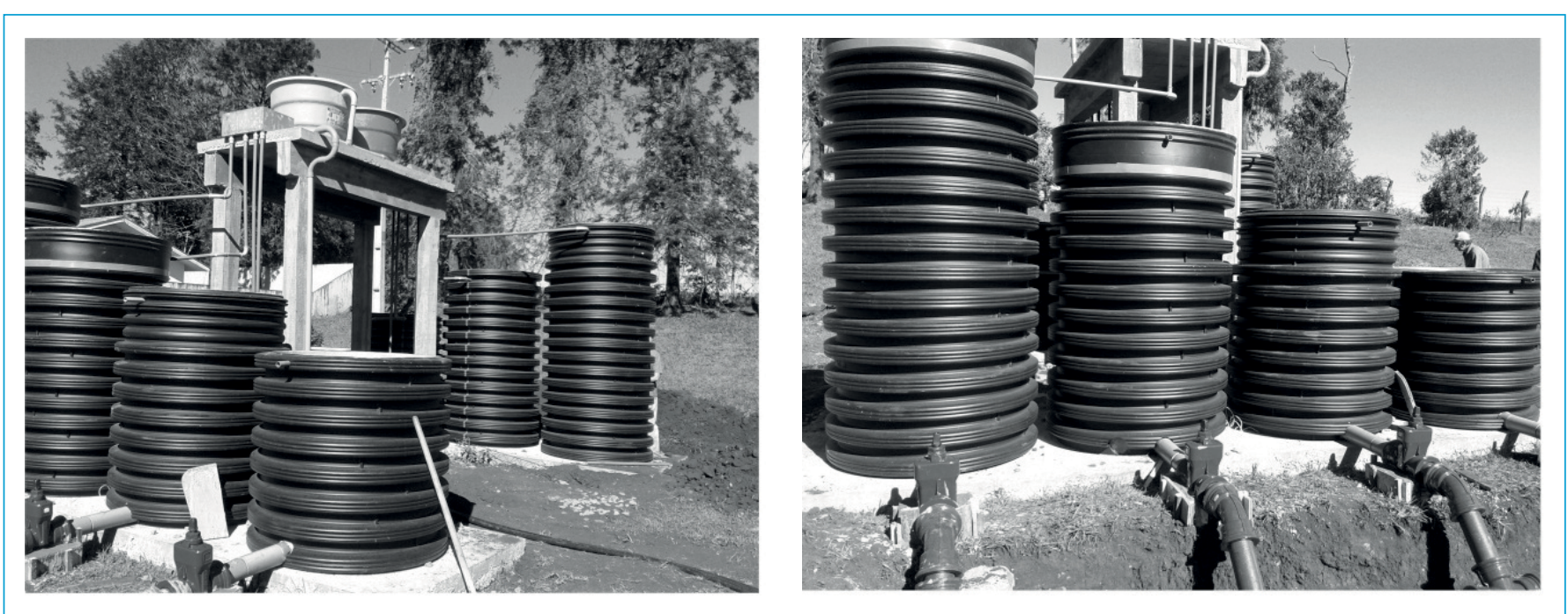

Figura 2 - Instalação experimental dos biofiltros submersos localizados na estação de tratamento de esgoto Norte da Companhia de Saneamento do Paraná em Cascavel (PR). 


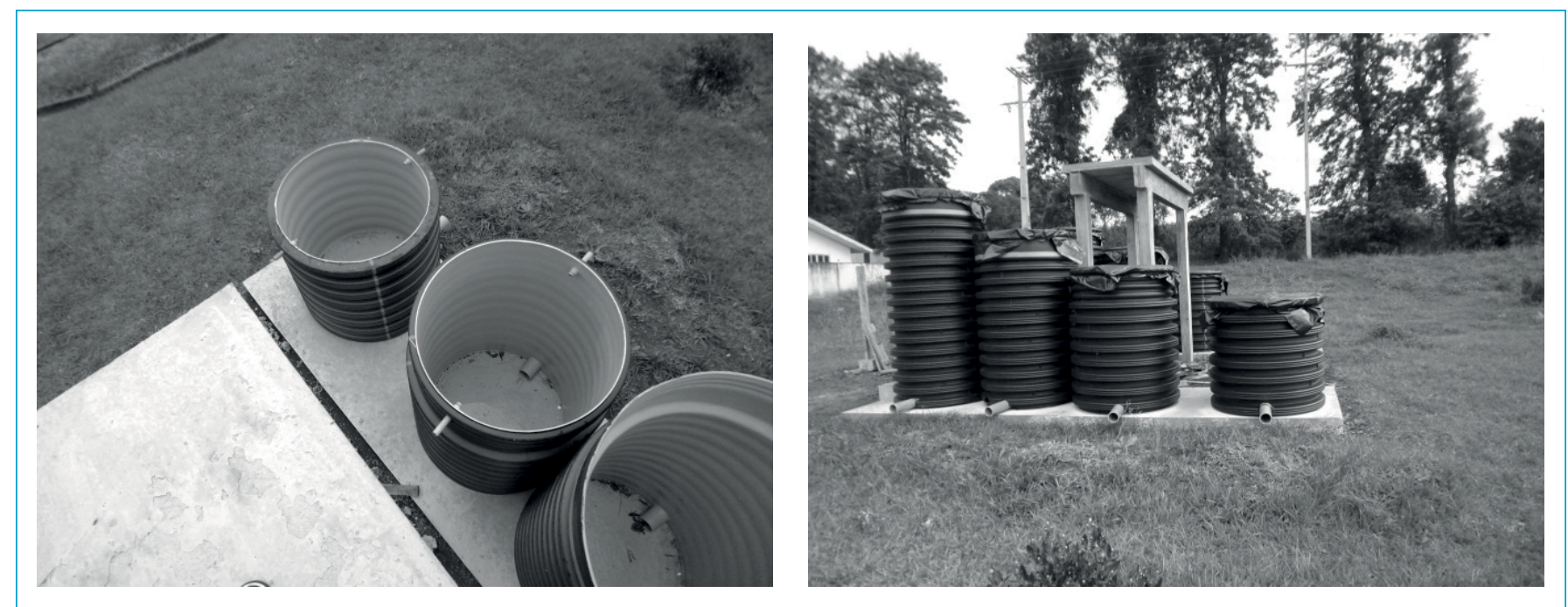

Figura 3 - Biofiltros submersos em polietileno de alta densidade com diâmetro nominal de 1.050 mm revestidos internamente com fibra de vidro.

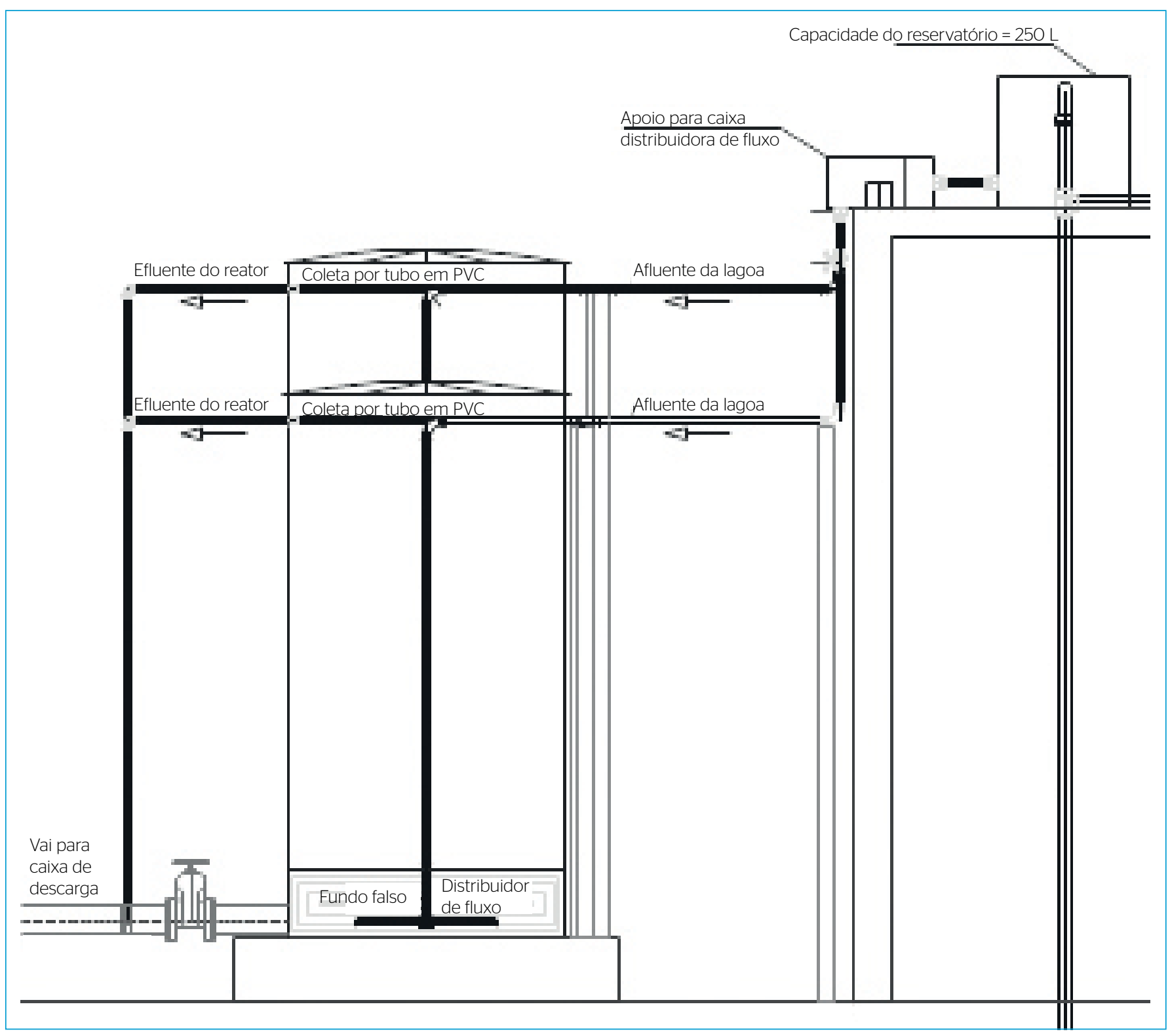

Figura 4 - Recheio dos biofiltros submersos (brita nº 3) e corte dos biofiltros mostrando seu fundo falso. 
O afluente dos biofiltros submersos era conduzido por um tubo de PVC com DN de $25 \mathrm{~mm}$, passando pelo interior do recheio, distribuindo-se o fluxo por uma malha do mesmo tubo, com furações de $\varnothing$ $=8 \mathrm{~mm}$. O fluxo ascendente ocorreu uniformemente no fundo falso em fibra de vidro reforçada, pelas furações nele executadas em malha de $10 \mathrm{~cm}$, com furos de $\varnothing=20 \mathrm{~mm}$.

Após passagem pelo leito filtrante do biofiltro submerso, três trechos de tubulações em PVC com DN de $25 \mathrm{~mm}$, com furos de $\varnothing=10 \mathrm{~mm}$, cada um com $3 \mathrm{~cm}$, coletavam, na superfície, o efluente final, de modo uniforme, buscando-se evitar caminhos preferenciais (Figura 5).

Os reservatórios de entrada, as caixas distribuidoras de vazão e os biofiltros submersos foram cobertos com tampas em fibra de vidro, conforme a Figura 6.

\section{Dimensionamento dos biofiltros submersos}

Para o estudo, foram consideradas duas zonas de captação na lagoa de maturação da ETE Norte em Cascavel (PR), a superficial (ZS) e a intermediária (ZI). Para cada uma delas, foram considerados, para o estudo, quatro biofiltros submersos, totalizando oito unidades. No dimensionamento dos biofiltros, foram observados os seguintes parâmetros e dados:

- diâmetro do biofiltro $=105 \mathrm{~cm}$;

- recheio em pedra brita $n^{\circ} 3$;

- alturas dos recheios variando entre 50, 100, 150 e $200 \mathrm{~cm}$;

- taxa de aplicação volumétrica de $0,50 \mathrm{~m}^{3} \cdot \mathrm{m}^{-3} \cdot \mathrm{d}^{-1}$ adotada com base em Sezerino et al (2005): 0,17 a 1,48 $\mathrm{m}^{3} \cdot \mathrm{m}^{-3} \cdot \mathrm{d}^{-1}$; Andrada (2005): 0,80 $\mathrm{m}^{3} \cdot \mathrm{m}^{-3} \cdot \mathrm{d}^{-1} ;$ Middlebrooks (1988): $0,80 \mathrm{~m}^{3} \cdot \mathrm{m}^{-3} \cdot \mathrm{d}^{-1} ; \mathrm{EPA}$ (1983): Eudora (Kansas) - até $1,20 \mathrm{~m}^{3} \cdot \mathrm{m}^{-3} \cdot \mathrm{d}^{-1}\left(0,40 \mathrm{~m}^{3} \cdot \mathrm{m}^{-3} \cdot \mathrm{d}^{-1}\right.$ no inverno) - Califórnia e Missouri - 0,25 e 0,40 $\mathrm{m}^{3} \cdot \mathrm{m}^{-3} \cdot \mathrm{d}^{-1}-\mathrm{e}$ Veneta (Oregon) $-0,3 \mathrm{~m}^{3} \cdot \mathrm{m}^{-3} \cdot \mathrm{d}^{-1}$.

Um fluxograma descritivo dos dez pontos de amostragem é apresentado a seguir na Figura 7.
Como resultado da taxa de aplicação volumétrica, a vazão varia segundo a altura do recheio, conforme apresentado nas Tabelas 3 e 4.

\section{RESULTADOS E DISCUSSÃO}

\section{Análise dos valores de clorofila $\mathrm{A}$}

Os resultados de clorofila A (Tabela 5) foram obtidos para os dez pontos de amostragem do SBS e, nas Tabelas 6 e 7 e nas Figuras 8 e 9, temos os valores dessa clorofila para a ZS e a ZI, separadamente. Na Tabela 8 e nas Figuras 10 e 11, são apresentados os dados de eficiência de remoção dessa variável.

Os resultados de clorofila A são correlacionados à quantidade de algas nos afluentes e efluentes dos biofiltros submersos do experimento. Os valores de eficiência de remoção dessa clorofila nos biofiltros submersos da zona intermediária situaram-se em valores mínimos de $3,80 \%$ e máximos de $98,30 \%$, conforme resultados contidos na Tabela 8 e na Figura 10.

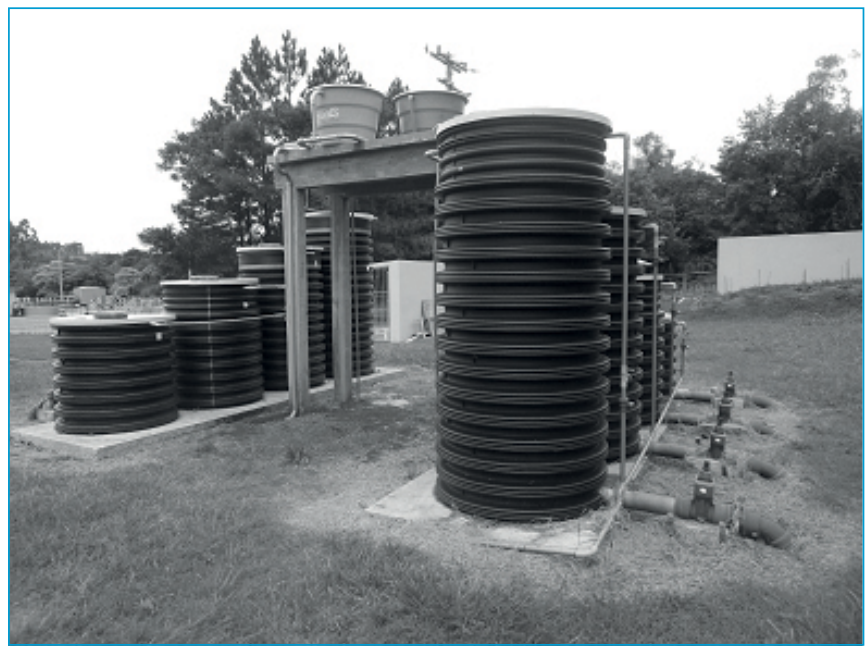

Figura 6 - Cobertura dos reservatórios, caixas distribuidoras de vazão e biofiltros submersos.

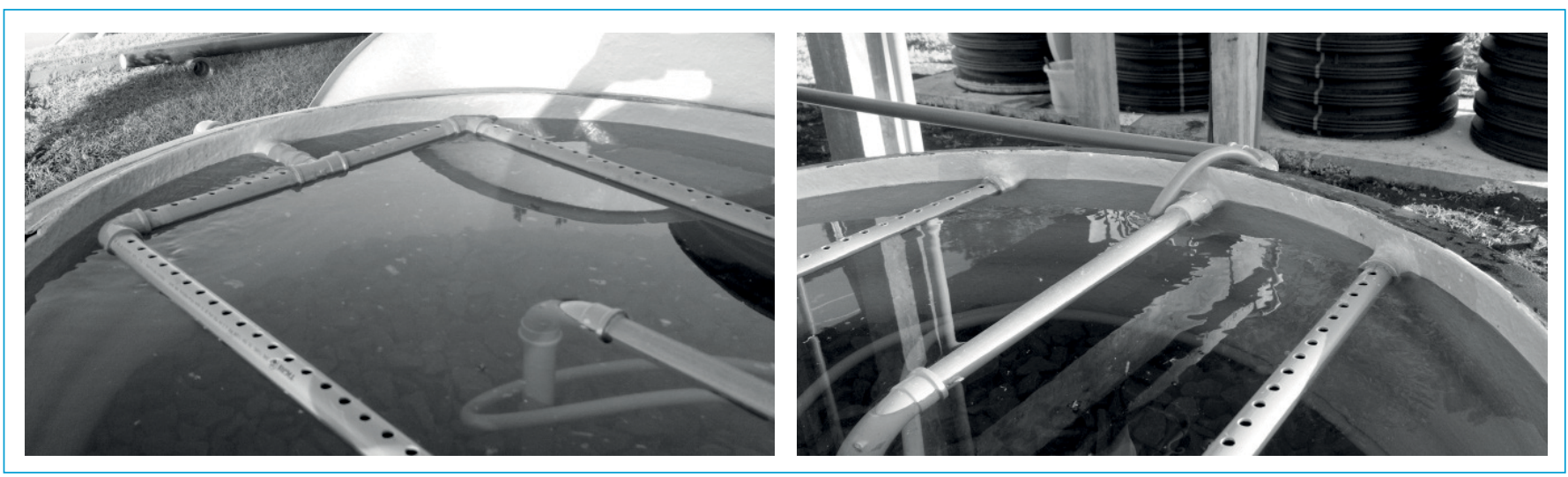

Figura 5 - Dispositivo de coleta superficial do efluente final em policloreto de polivinila com diâmetro nominal de $25 \mathrm{~mm}$. 
Para os biofiltros operando com afluente da ZS, o valor mínimo foi de $65,70 \%$ e o máximo de $98,10 \%$, sendo a média de $84,45 \%$, conforme Tabela 8 e Figura 11.
Assim, pode-se concluir que a remoção de clorofila A para os biofiltros submersos da ZS foi mais efetiva. Para os valores médios de eficiência de remoção de clorofila $\mathrm{A}$, os maiores

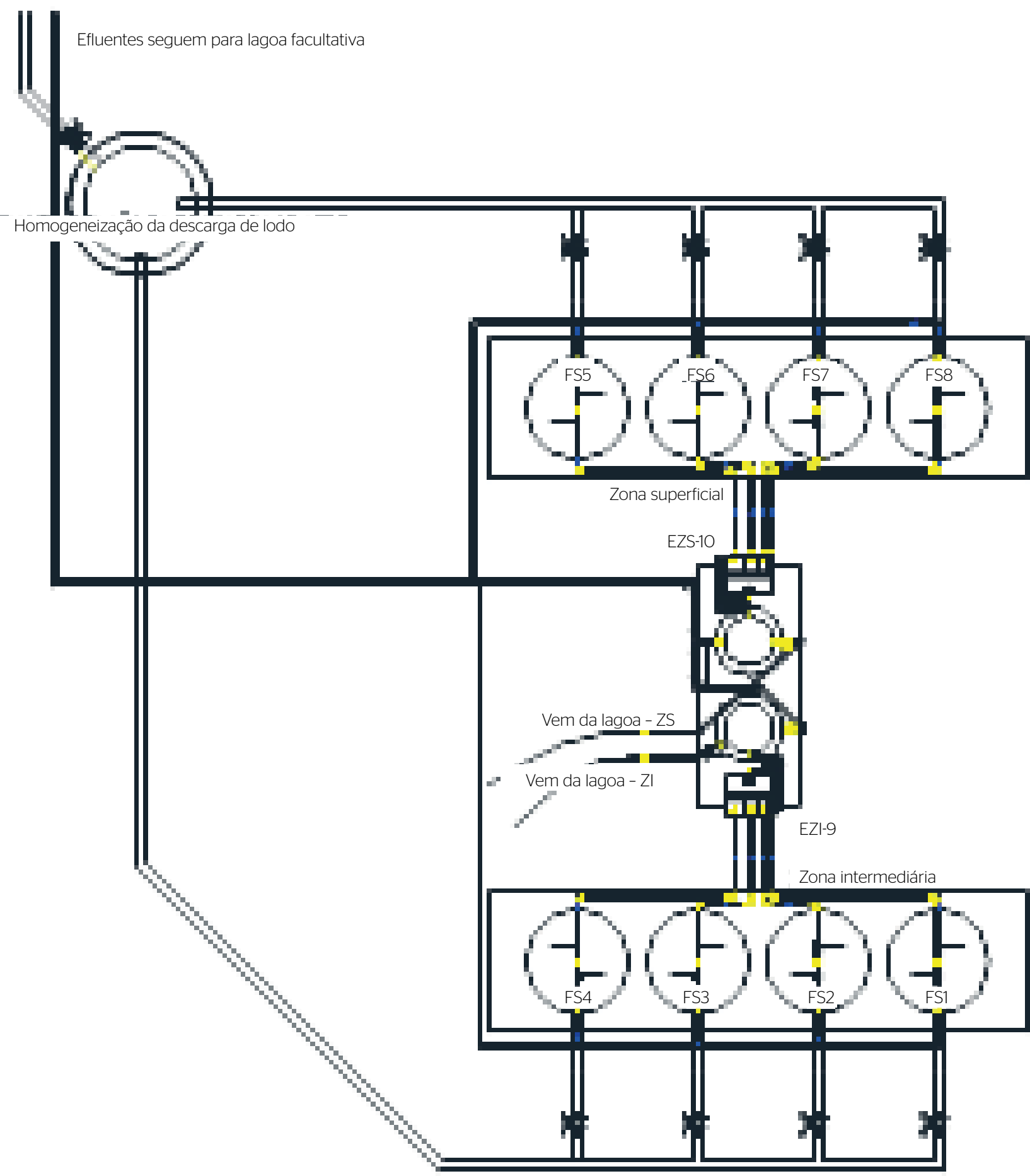

FS: biofiltro submerso; EZS: entrada da zona superficial; ZS: zona superficial; ZI: zona intermediária; EZI: entrada da zona intermediária.

Figura 7 - Sistema de biofiltros submersos, mostrando a numeração dos pontos de coleta de amostras: 1 a 8 para os biofiltros submersos (de 1 a 4 para a zona intermediária e de 5 a 8 para a zona superficial), 9 para a entrada da zona intermediária e 10 para entrada da zona superficial. 
Tabela 3 - Volume útil e volume dos recheios utilizados nos biofiltros submersos.

\begin{tabular}{l|c|c}
\multicolumn{3}{l}{ Volumes de biofiltros submersos } \\
\hline Biofiltro & Volume vazio (m3) & Volume de pedra (m3) \\
\hline 1 & 0,866 & 0,433 \\
\hline 2 & 1,299 & 0,866 \\
\hline 3 & 1,732 & 1,299 \\
\hline 4 & 2,165 & 1,732 \\
\hline 5 & 0,866 & 0,433 \\
\hline 6 & 1,299 & 0,866 \\
\hline 7 & 1,732 & 1,299 \\
\hline 8 & 2,165 & 1,732 \\
\hline
\end{tabular}

Tabela 4 - Vazão volumétrica segundo o volume do recheio dos biofiltros submersos.

\begin{tabular}{|c|c|c|c|c|c|c|}
\hline \multicolumn{4}{|c|}{ Unidades de bombeamento } & \multicolumn{3}{|c|}{ Qtotal = 90 L.h-1 } \\
\hline Taxas & & 0,5 & m3.m-3.d-1 & $h=$ & 0,5 & $\mathrm{~m}$ \\
\hline Diâmetro & Área & Volume & Vazão & \multicolumn{3}{|c|}{ Vazão } \\
\hline $\mathrm{m}$ & $\mathrm{m} 2$ & m3.m-1 & m3.d-1 & \multicolumn{3}{|c|}{ L.h-1 } \\
\hline 1,05 & 0,8659 & 0,4329 & 0,2165 & \multicolumn{3}{|c|}{9,0195} \\
\hline Taxas & & 0,5 & m3.m-3.d-1 & $h=$ & 1,0 & $\mathrm{~m}$ \\
\hline Diâmetro & Área & Volume & Vazão & \multicolumn{3}{|c|}{ Vazão } \\
\hline $\mathrm{m}$ & $\mathrm{m} 2$ & m3.m-1 & m3.d-1 & \multicolumn{3}{|c|}{ L.h-1 } \\
\hline 1,05 & 0,8659 & 0,8659 & 0,4329 & \multicolumn{3}{|c|}{18,0391} \\
\hline Taxas & & 0,5 & m3.m-3.d-1 & $h=$ & 0,5 & $\mathrm{~m}$ \\
\hline Diâmetro & Área & Volume & Vazão & \multicolumn{3}{|c|}{ Vazão } \\
\hline $\mathrm{m}$ & $\mathrm{m} 2$ & m3.m-1 & m3.d-1 & \multicolumn{3}{|c|}{ L.h-1 } \\
\hline 1,05 & 0,8659 & 1,2988 & 0,6494 & \multicolumn{3}{|c|}{27,0586} \\
\hline Taxas & & 0,5 & m3.m-3.d-1 & $h=$ & 0,5 & $\mathrm{~m}$ \\
\hline Diâmetro & Área & Volume & Vazão & \multicolumn{3}{|c|}{ Vazão } \\
\hline $\mathrm{m}$ & $\mathrm{m} 2$ & m3.m-1 & m3.d-1 & \multicolumn{3}{|c|}{ L.h-1 } \\
\hline 1,05 & 0,8659 & 1,7318 & 0,8659 & \multicolumn{3}{|c|}{36,0782} \\
\hline
\end{tabular}

Tabela 6 - Valores de clorofila A, em $\mu \mathrm{g} \cdot \mathrm{L}^{-1}$, para os biofiltros submersos alimentados com afluente extraído da zona intermediária da lagoa de maturação.

\begin{tabular}{|c|c|c|c|c|c|c|}
\hline & & \multicolumn{5}{|c|}{ Pontos de coleta } \\
\hline & & BS-1 & BS-2 & BS-3 & BS-4 & EZI \\
\hline \multirow{2}{*}{2013} & 15/dez. & 60,84 & 4,6 & 24,67 & 46,04 & 296,99 \\
\hline & 30/dez. & 23,02 & 48,68 & 63,80 & 36,18 & 231,87 \\
\hline \multirow{7}{*}{2014} & 15/jan. & 165,43 & 110,18 & 124,32 & 112,15 & 948,19 \\
\hline & 30/jan. & 52,95 & 28,28 & 97,02 & 39,47 & 455,84 \\
\hline & 15/fev. & 4,6 & 5,92 & 7,89 & 2,3 & 57,23 \\
\hline & 07/mar. & 59,84 & 69,64 & 124,85 & 102,31 & 435,21 \\
\hline & 16/abr. & 250,12 & 159,84 & 307,84 & 61,67 & 357,17 \\
\hline & 30/abr. & 37,00 & 32,56 & 63,64 & 44,40 & 334,48 \\
\hline & 15/mai. & 79,43 & 68,08 & 87,81 & 118,4 & 542,67 \\
\hline
\end{tabular}

BS: biofiltro submerso; EZl: entrada da zona intermediária.

Tabela 7 - Valores de clorofila A, em $\mu \mathrm{g} \cdot \mathrm{L}^{-1}$, para os biofiltros submersos alimentados com afluente extraído da zona superficial da lagoa de maturação.

\begin{tabular}{|c|c|c|c|c|c|c|}
\hline & \multicolumn{5}{|c|}{ Pontos de coleta } \\
\hline & & BS-5 & BS-6 & BS-7 & BS-8 & EZS \\
\hline \multirow{2}{*}{2013} & 15/dez. & 16,12 & 6,91 & 7,89 & 89,46 & 361,12 \\
\hline & 30/dez. & 75,64 & 34,2 & 26,97 & 56,24 & 227,92 \\
\hline \multirow{7}{*}{2014} & 15/jan. & 73,67 & 68,08 & 27,63 & 20,39 & 758,75 \\
\hline & 30/jan. & 87,48 & 90,77 & 112,48 & 45,39 & 557,47 \\
\hline & 15/fev. & 33,55 & 17,43 & 1,97 & 1,32 & 295,01 \\
\hline & 07/mar. & 60,43 & 70,21 & 66,35 & 62,65 & 235,41 \\
\hline & 16/abr. & 145,53 & 174,15 & 185,49 & 144,05 & 540,69 \\
\hline & $30 / a b r$. & 43,41 & 56,73 & 46,87 & 39,96 & 336,45 \\
\hline & 15/mai. & 126,79 & 169,21 & 62,65 & 118,89 & 580,56 \\
\hline
\end{tabular}

BS: biofiltro submerso; EZS: entrada da zona superficial.

Tabela 5 - Valores de clorofila A em $\mu \mathrm{g} \cdot \mathrm{L}^{-1}$ nos 10 pontos do sistema de biofiltros submersos.

\begin{tabular}{|c|c|c|c|c|c|c|c|c|c|c|c|}
\hline & & \multicolumn{10}{|c|}{ Pontos de coleta } \\
\hline & & BS-1 & BS-2 & BS-3 & BS-4 & BS-5 & BS-6 & BS-7 & BS-8 & $\mathrm{EZI}$ & EZS \\
\hline \multirow{2}{*}{2013} & 15/dez. & 60,84 & 4,6 & 24,67 & 46,04 & 16,12 & 6,91 & 7,89 & 89,46 & 296,99 & 361,12 \\
\hline & 30/dez. & 23,02 & 48,68 & 63,80 & 36,18 & 75,64 & 34,2 & 26,97 & 56,24 & 231,87 & 227,92 \\
\hline \multirow{7}{*}{2014} & 15/jan. & 165,43 & 110,18 & 124,32 & 112,15 & 73,67 & 68,08 & 27,63 & 20,39 & 948,19 & 758,75 \\
\hline & 30/jan. & 52,95 & 28,28 & 97,02 & 39,47 & 87,48 & 90,77 & 112,48 & 45,39 & 455,84 & 557,47 \\
\hline & 15/fev. & 4,6 & 5,92 & 7,89 & 2,3 & 33,55 & 17,43 & 1,97 & 1,32 & 57,23 & 295,01 \\
\hline & 07/mar. & 59,84 & 69,64 & 124,85 & 102,31 & 60,43 & 70,21 & 66,35 & 62,65 & 435,21 & 235,41 \\
\hline & 16/abr. & 250,12 & 159,84 & 307,84 & 61,67 & 145,53 & 174,15 & 185,49 & 144,05 & 357,17 & 540,69 \\
\hline & 30/abr. & 37,00 & 32,56 & 63,64 & 44,40 & 43,41 & 56,73 & 46,87 & 39,96 & 334,48 & 336,45 \\
\hline & 15/mai. & 79,43 & 68,08 & 87,81 & 118,4 & 126,79 & 169,21 & 62,65 & 118,89 & 542,67 & 580,56 \\
\hline
\end{tabular}

BS: biofiltro submerso; EZS: entrada da zona superficial; EZI: entrada da zona intermediária. 
valores foram dos biofiltros submersos 7 e $8(150$ e $200 \mathrm{~cm}$, respectivamente), com os valores de 86,00 e $87,30 \%$, respectivamente, na $Z S$.

\section{CONCLUSÕES}

$\mathrm{Na}$ avaliação da influência da profundidade de tomada de afluente de acordo com a zona lagunar (intermediária $-180 \mathrm{~cm}-\mathrm{e}$

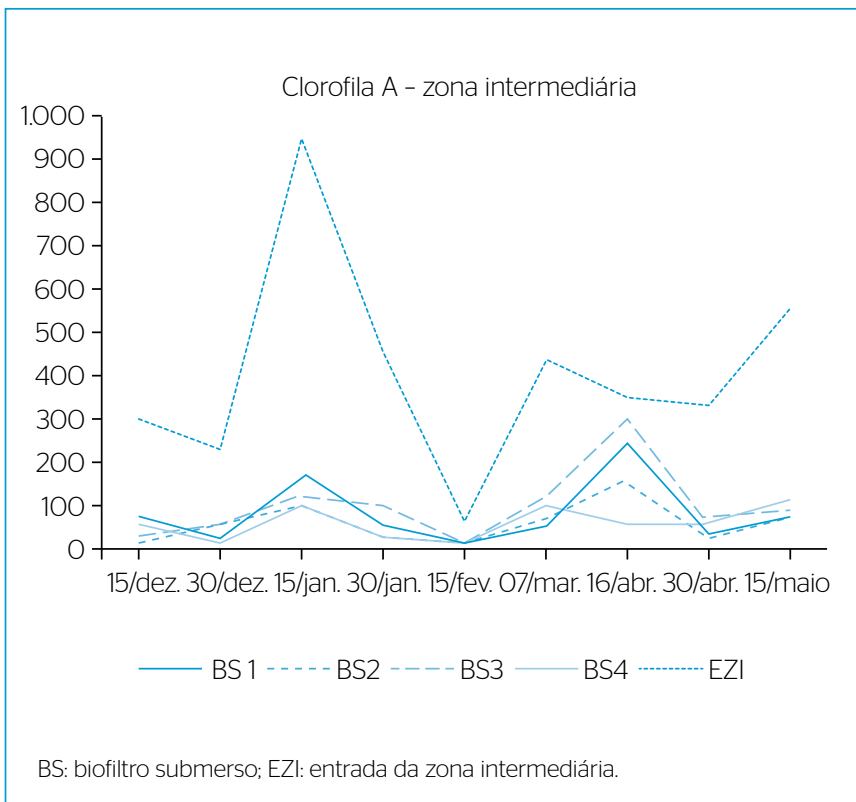

Figura 8 - Valores de clorofila A em $\mu \mathrm{g} \cdot \mathrm{L}^{-1}$, obtidos para os biofiltros submersos alimentados com afluente extraído da zona intermediária da lagoa de maturação.

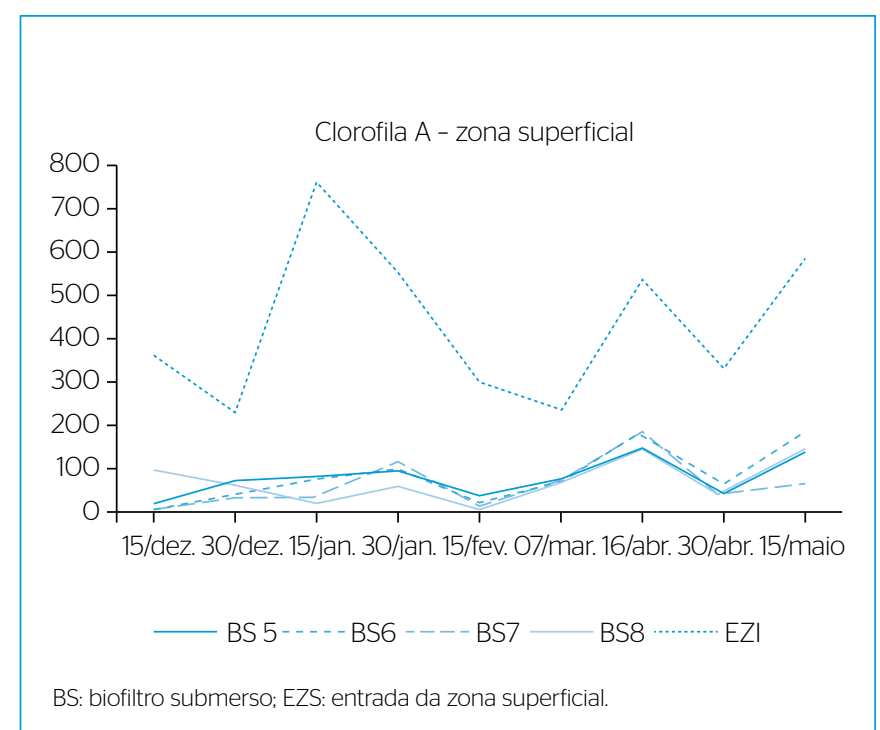

Figura 9 - Valores de clorofila A, em $\mu \mathrm{g} . \mathrm{L}^{-1}$, obtidos para os biofiltros submersos alimentados com afluente extraído da zona superficial da lagoa de maturação.
Tabela 8 - Valores percentuais de eficiências de remoção mínima, média e máxima para clorofila $A$.

\section{Eficiência de remoção da clorofila A}

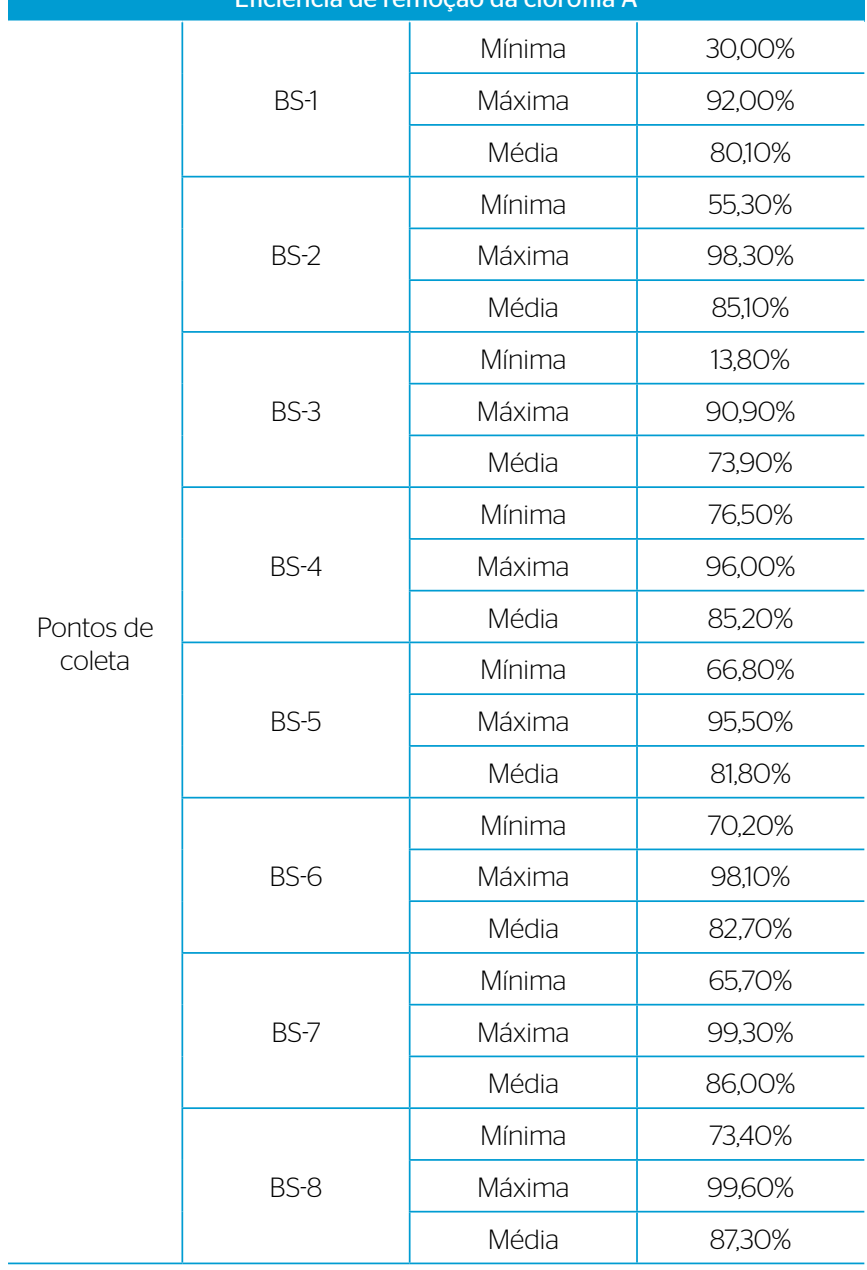

BS: biofiltro submerso.

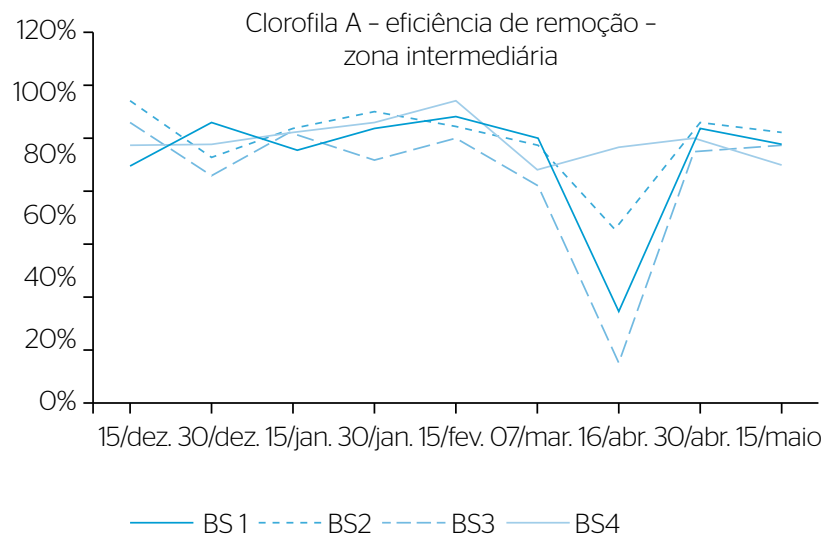

BS: biofiltro submerso

Figura 10 - Valores percentuais de eficiência de remoção de clorofila A na zona intermediária. 


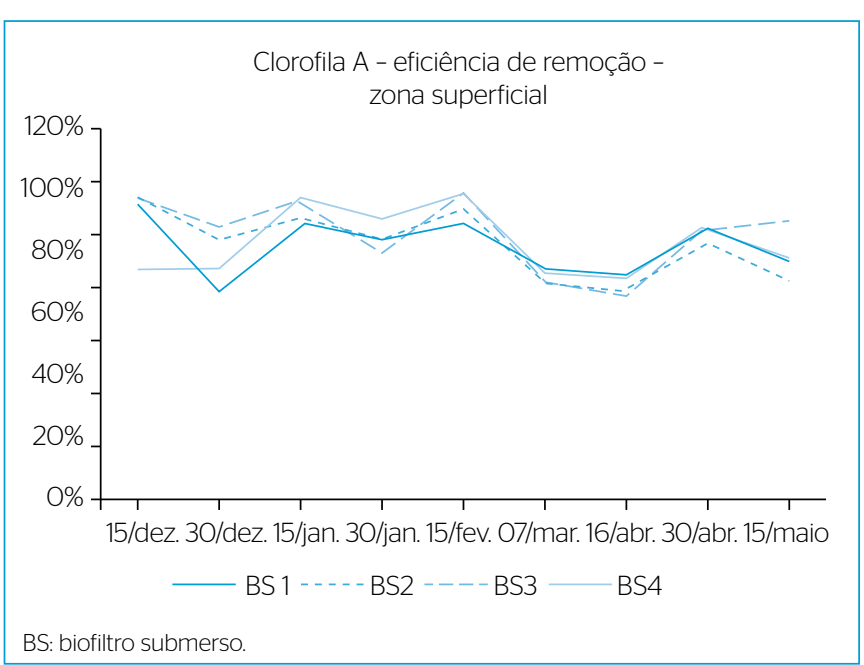

Figura 11 - Valores percentuais de eficiência de remoção de clorofila A na zona superficial. superficial $-60 \mathrm{~cm}$ ) sobre a eficiência de remoção das algas, constatou-se o que parece ser uma tendência geral: a tomada de afluente na ZS mostrou-se como a melhor alternativa.

Já na avaliação da influência da altura da camada filtrante de pedra brita sobre a eficiência de remoção das algas, foi observado que as camadas de maior espessura (150 e $200 \mathrm{~cm}$ ) apresentaram os melhores resultados, com uma tendência de valores mais constantes e uniformes, para a mesma taxa de aplicação volumétrica.

Essa alternativa de pós-tratamento (SBS) exposta pela pesquisa pareceu demonstrar viabilidade de aplicação, atendendo aos requisitos da atual legislação ambiental, já que tem uma concepção de baixo custo por não necessitar de fonte de energia externa bem como por apresentar operação e manutenção simples e não dispendiosas quando comparada a outros sistemas.

\section{REFERÊNCIAS}

AGÊNCIA DE PROTEÇÃO AMBIENTAL DOS ESTADOS UNIDOS (USEPA). (1983) Design Manual - Municipal Wastewater Stabilization Ponds. U.S. Environmental Protection Agency. EPA-625/1-83015. 327 p.

ANDRADA, J.G.B. (2005) Utilização de Filtros Grosseiros para Remoção de Algas presentes em Efluentes de lagoas de Polimento. $115 f$. Dissertação (Mestrado em Saneamento, Meio Ambiente e Recursos Hídricos) - Universidade Federal de Minas Gerais, Belo Horizonte.
MIDDLEBROOKS, E.J. (1988) Review of rock filters for the upgrade of lagoon effluents. Journal WPCF, v. 60, n. 9, p. 16571662.

YEH, T.Y.; KE, T.Y.; LIN, Y.L. (2011) Algal Growth Control Within Natural Water Purification Systems: Macrophyte Light Shading Effects. Water Air Soil Pollution, v. 214, n. 1, p. 575-586. http://doi.org/10.1007/ s11270-010-0447-4 